\title{
Frontal lobe cerebral aneurysm rupture presenting as psychosis
}

\author{
Donald P Hall, Stephen A Young
}

\begin{abstract}
A 23 year old male with acute onset of blunted affect, looseness of associations and auditory hallucinations presented to $a$ tertiary care hospital 10 days after development of symptoms. Before transfer, the patient received a diagnosis of schizophreniform disorder and treatment with haloperidol was started which resulted in moderate improvement. Examination led to detection of a ruptured cerebral aneurysm in the left frontal lobe. Evacuation of the haematoma and repair of the aneurysm resulted in nearly complete resolution of symptoms. The rare incidence of acute aneurysm rupture, presenting in the case described, demonstrates the importance of a complete neurological examination in the evaluation of acute mental status changes.
\end{abstract}

(F Neurol Neurosurg Psychiatry 1992;55:1207-1208)

Vulnerability to depression associated with cerebral vascular disease has been noted since the time of Kraepelin. ${ }^{1}$ However, the association of psychotic symptoms have been rarely reported. Delusional symptoms have been occasionally associated with right hemisphere infarcts. ${ }^{2}$ Acute confusional states, which may be related to delusional processes, have also been associated with cerebrovascular disease. ${ }^{34}$ Two case reports describe right hemisphere infarcts presenting with paranoid delusions and hallucinations. ${ }^{56}$ We found no reports of an association between psychosis and cerebral artery aneurysm.

\section{Case history}

Ten days before admission, a 23 year old soldier was taken to the emergency room of an overseas hospital with the chief complaint of a recent onset of difficulty awakening from sleep and unusual behaviour. There was a previous history of migraine headaches which had been treated with isometheptene three months earlier. Further history was limited by the patient's grossly disorganised behaviour. Contact with coworkers revealed no recent deterioration in performance, mood or thought processes. Mental status examination revealed a sedate, disoriented patient with blunted affect, looseness of associations, paranoid ideation and auditory hallucinations. Physical examination, serum chemistries, urine drug testing (including amphetamine and phencyclidine), and EEG were all normal. A provisional diagnosis of schizophreniform disorder was made and a regime of haloperidol $(20 \mathrm{mg} /$ day) and benztropine $(2 \mathrm{mg} /$ day $)$ was started. The patient's mental status improved over the next week.

Mental status examination at the time of admission to our unit revealed a dishevelled and fatigued young man who was disoriented to time and place. He showed a relaxed and sedate mood with a constricted range of affect. Thought processes were loosely associated. Thought content was remarkable for paranoid ideation, yet the patient refused to give details. He denied auditory or visual hallucinations when interviewed by one of the authors (DH), but, affirmed the presence of these when questioned by another physician. $\mathrm{He}$ also reported to a nurse that he saw an image on a disconnected video screen. Evidence of responding to internal stimuli, including darting eye movements, were also observed. He was able to recall one out of three objects at three minutes and remembered the three most recent presidents of the United States. Serial seven subtraction examination produced the following response: " $100-85-97$-the ultimate-you know-the ultimate-we all have it". When asked the similarity of a bicycle and a bus, he replied: "one is loud and one isn't, two wheels". Physical and detailed neurological examination was normal, with the exception of papilloedema, which was worse on the right. Computed axial tomography revealed a $4 \mathrm{~cm}$ hyperdense mass with a ring of enhancement in the left frontal lobe, evidence of blood in the sylvian fissure, and significant mass effect with shift of the midline structures (figure).

\section{Neurosurgical intervention}

Arteriography showed a $1.2 \mathrm{~cm}$ middle cerebral artery aneurysm. Left pterional craniotomy showed a $1.2 \mathrm{~cm}$ saccular aneurysm and $7 \mathrm{~cm}$ frontal haematoma. The aneurysm was ligated, the frontal haematoma evacuated, and the temporal lobe partially resected. The surgery was complicated by intraoperative rupture of the aneurysm, and transient postoperative hemiparesis and aphasia. Six weeks after surgery the patient was oriented in all spheres, demonstrated a blunted range of affect, completed serial seven subtractions correctly, and 
Figure 1 Computerised axial tomography scan with contrast revealing a $4 \mathrm{~cm}$ hyperdense mass with ring of enhancement, representing a large middle cerebral aneurysm and haematoma in the left frontal lobe.

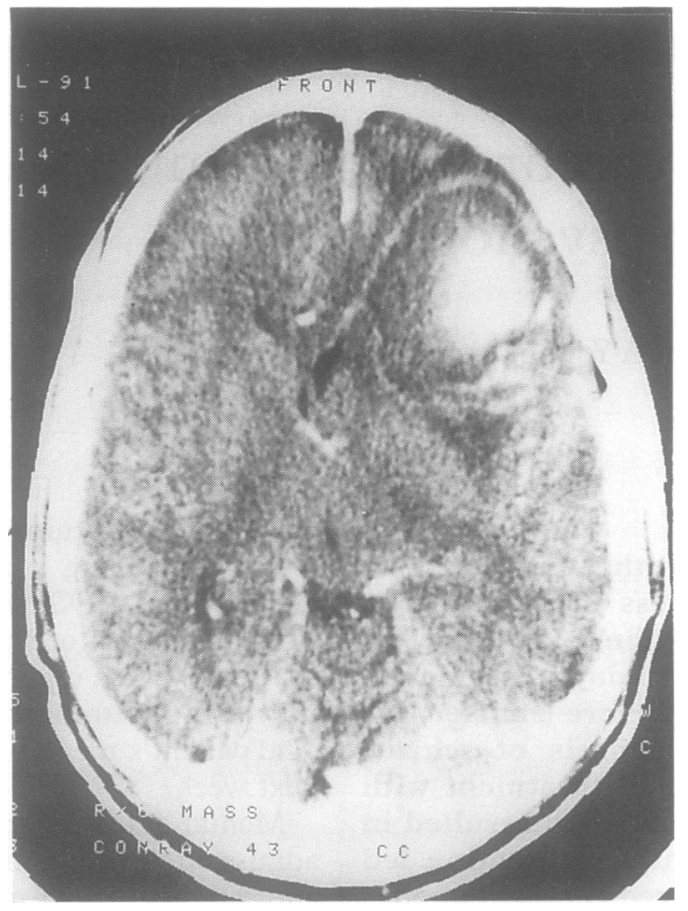

showed infrequent brief looseness of associations (noted only upon awakening). Formal neuropsychiatric testing, three months after surgery, revealed mild residual aphasia (expressive $>$ receptive) and moderate deficits in memory skills. He was returned to independent community living.

\section{Discussion}

Symptoms of a thought disorder, a normal preliminary medical and neurological evaluation, and therapeutic response to neuroleptic medication at a referring hospital removed most suspicions of an anatomical disease. The findings of papilloedema and a frontal lobe lesion, however, serve as a cautionary statement regarding the evaluation of psychiatric illnesses. This patient also broadens the clinical presentation of cerebral artery aneurysm rupture.

There are relatively few reports of psychosis associated with cerebrovascular disease. Primary literature describing psychosis associated with acute localised changes in the brain has resulted from brain tumour and epilepsy research. A large review by Davison and Bagley $^{7}$ reported a statistically significant correlation between temporal lobe masses and psychosis. Frontal lobe tumours did not correlate to a significant level. Historically, the temporal lobe has been a focus for a number of disorders that present with behavioural symptoms. Gibbs and Gibbs reported that $0.3 \%$ of 296 patients with a midtemporal spike focus demonstrated psychosis, compared with $10 \%$ of 169 patients with an anterior temporal lobe focus. $^{8}$ Although this patient had a normal EEG, the presence of blood adjacent to the temporal lobe and the moderate mass effect make it difficult to exclude a temporal lobe seizure in this patient. Additionally, temporal lobe activity is difficult to assess with standard EEG techniques.

Although much attention has been directed towards the association between temporal pathology and psychosis, recent functional brain imaging studies have found abnormalities in the frontal lobes of schizophrenics. Buchsbaum $^{9}$ has reviewed PET studies of the frontal lobes of schizophrenic patients and reports wide replication of reduced frontal lobe function findings. One study has shown a specific area in the left prefrontal cortex which has decreased metabolic activity in schizophrenia. ${ }^{10}$ It is remarkable that massive frontal lobe lesions often remain silent and/or produce minor changes in behaviour. A number of physiological and electrical interpretations can be offered to explain the syndrome of psychosis demonstrated by this patient. Patients with focal lesions who present with acute confusional states have been described elsewhere, but such patients generally have findings more consistent with an acute delirium (for example, shifting consciousness). Physiological changes which may have contributed to this syndrome include diffuse left cerebral ischaemia, and those produced by the extensive extravasation of blood and parenchymal destruction or compression. The possibility of the development of seizure foci due to these changes must also be considered. However, the location of the lesion as well as the clinical findings (lack of automatisms, discrete episodes, and ictal/ post ictal confusion) make the diagnosis of complex partial seizures unlikely.

Perhaps one of the most intriguing interpretations may be that this patient's psychosis resulted from local changes in metabolic function of the left prefrontal cortex, which resolved following neurosurgical repair of surrounding tissues.

1 Kraepelin E. Psychiatrie, ein Lehrbuch der Psychiatrie. Leipzig, 1904. (Translated by AR Diefendorf as Clinical psychiatry). New York: MacMillan, 1915

2 Levine DN, Grek A. The anatomic basis of delusions after right cerebral infarction. Neurology 1984;34:577-82.

3 Mesulam M, Waxman SG, Geschwind N, et al. Acute confusional states with right cerebral artery infarctions. $\mathcal{f}$ Neurol Neurosurg Psychiatry 1976;39:84-9.

4 Price BH, Mesulam M. Psychiatric manifestations of right hemisphere infarctions. $\mathcal{f}$ Nerv Ment Dis 1985;173: 610-4.

5 Peroutka SJ, Sohmer BH, Kumar AJ, et al. Hallucinations and delusions following a right temporoparietooccipital infarction. The fohns Hopkins Medical fournal 1982; infarction.

6 Berthier M, Starkstein S. Acute atypical psychosis following right hemiphere stroke. Acta Neurol Belg 1987;87: right hemis

7 Davison K, Bagley CR. Schizophrenia-like psychoses associated with organic disorders of the central nervous system. In: Herrington RN, ed. Current problems in neuropsychiatry. Kent: Headley 1969.

8 Gibbs FA, Gibbs EL. Atlas of electroencephalography, vol 2: epilepsy. Cambridge, Mass: Addison Wesley Press, 1952.

9 Buchsbaum MS. Positron emission tomography and regional brain metabolism in schizophrenia research. In: Volkow ND, Wolf AP, eds. Positron emission tomography in schizophrenia research, Vol 33. Washington: American Psychiatry Press, 1991.

10 Cohen RM, Semple WE, Gross M, et al. Dysfunction in a prefrontal substrate of sustained attention in schizophrenia. Life Sci 1987:2031-9. 